\title{
Antifungal agents: Polyene, azole, antimetabolite, other and future agents
}

\author{
Fardin Ali Malayeri ${ }^{1}$, Ali Akbar Rezaei ${ }^{2}$, Omid Raiesi ${ }^{3 *}$
}

1. Department of Clinical Biochemistry, Faculty of Medicine, Zabol University of Medical sciences, Zabol, Iran

2. Department of Pathobiology, Faculty of Veterinary Medicine, Shahid Bahonar University of Kerman, Kerman, Iran

3. Department of Medical Parasitology and Mycology, School of Public Health, Tehran University of Medical Sciences, Tehran, Iran

*Corresponding author:Tel: +98 9189428310 Fax: +98 8433622946

Address: Department of Medical Parasitology and Mycology, School of Public Health, Tehran University of

Medical Sciences, Tehran, Iran

E-mail: omid_raissi69@yahoo.com

Received; 2017/12/30 revised; 2018/03/13 accepted; 2018/04/14

\begin{abstract}
Antifungals have always been considered as one of the astonishing discoveries of the 20th century. This is correct, but the real marvel is the development of antifungal resistance in hospitals, communities, and the environment concomitant with their use. Fungal infections have emerged as an important clinical threat, with significant associated morbidity and mortality. This study is designed to provide a comprehensive view of antifungal agents and related agents. Information was based on the expertise of some literatures. Over the past decades, the incidence and diversity of fungal infection has grown in association with an increasing number of immunocompromised patients. An understanding of the pharmacokinetic and pharmacodynamics properties of the classes of antifungal compounds is vital for the effective management of invasive fungal infections. This review provides a summary of the pharmacologic principles involved in treatment of fungal diseases. Clinical needs for novel antifungal agents have altered steadily with the rise and fall of AIDS-related mycoses, and the change in spectrum of fatal disseminated fungal infections that has accompanied change in therapeutic immunosuppressive therapies.
\end{abstract}

Keywords: Antifungal agents, Future agents, Polyenes, Azole

\section{Introduction}

The development of an antifungal agents has lagged behind that of antibacterial agents. This is a predictable consequence of the cellular structure of the organisms involved. Bacteria are prokaryotic and hence offer numerous structural and metabolic targets that differ from those of the human host. Fungi, in contrast, are eukaryotes, and consequently most agent toxic to fungi are also toxic to the host. Furthermore, because fungi generally grow slowly and often in multicellular forms, they are more difficult to quantify than bacteria. This difficulty complicates experiments designed to evaluate the in vitro or in vivo properties of a potential antifungal agents (1). Despite these limitations, numerous advances have been made in developing new antifungal agents and in understanding the existing ones. Three groups of drugs are emphasized; the polyenes, the azoles, and antimetabolite.

Copyright (C) 2018 Journal of Basic Research in Medical Science. This is an open access article distributed under the terms of the Creative Commons Attribution 4.0 International License (https://creativecommons.org/licenses/by/4.0/) which permits copy and redistribute the material, in any medium or format, provided the original work is properly cited. 
The increased use of antibacterial and antifungal agents in recent years has resulted in the development of resistance to these drugs (2).

\section{Early treatment}

Antifungal therapies evolved slowly during the early years of the past century. Potassium iodide was the standard treatment for cutaneous fungal infections including actinomycosis, blastomycosis, sporotrichosis, and tinea form the beginning of the $20^{\text {th }}$ century until after the Second World War (3).

First derived from sea algae, potassium iodide was considered to exert a direct antifungal effect, although the complete mechanism of action remains unclear $(4,5)$. Contemporarily, radiation was used to treat severe tinea captis infections, often with significant complicate ions including skin cancer and brain tumors. In the 1940s, Mayer et al, Demonstrated that sulfonamide drugs, such as sulfadiazine, exhibited both fungistatic and fungicidal activities against Histoplasma capsulatum (6, 7).This discovery led to the formation and the use of sulfonamide derivative for the treatment of blastomycosis, nocardiosis, and cryptococcosis. Griseofulvin, a compound derived from Penicillium griseofulvum, has been widely used to treat superficial fungal infections since its isolation in 1939(8-10). An understanding of the mechanisms of action and in vitro profiles of antifungal agents is pivotal to selecting effective treatments for dermatophytosis. The principal mechanisms of action of antifungal drugs include disruption of spindle and cytoplasmic microtubule function (e.g., griseofulvin), depletion of or binding to ergosterol (e.g., terbinafine, ketoconazole, and amphotericin B), and accumulation of squalene (terbinafine). It is likely that antifungal agents that deplete or bind to ergosterol have fungistatic activity only; agents that produce a concomitant accumulation of intracellular squalene have fungicidal activity. Although the mechanism of action markedly influences the clinical efficacy of an antifungal agent, in vitro and in vivo antimycotic profiles and bioavailability factors such as drug access to the stratum corneum also contribute to the effectiveness of antifungal agents (11). Meanwhile, six new antifungal agents have just reached, or are approaching, the clinic. Three are new triazoles, with extremely broad antifungal spectra, and three are echinocandins, which inhibit synthesis of fungal cell wall polysaccharides - a new mode of action. In addition, the sordarins represent a novel class of agents that inhibit fungal protein synthesis. This review describes the targets and mechanisms of action of all classes of antifungal agents in clinical use or with clinical potential (12) . In 1985, Gentle reported the successful treatment of ringworm in guinea pigs using oral griseofulvin, the successful attempts to develop novel and effective antifungal drugs encouraged the further study and discovery of new agents (13).

\section{Polyenes}

Nystatin, amphotericin B, pimaricin, are polyenes drugs that bind to sterol components in the phospholipid-sterol membranes of fungal cells to form complexes that induce physical changes in the membrane. The number of conjugated bonds and the molecular size of a particular polyene macrolide influence its affinity for different sterols in fungal cell membranes. Amphotericin B has a greater affinity for fungal ergosterol, the major sterol in fungal membranes, than for eukaryotic (host) cell membrane cholesterol (14). The long polyene structure causes the formation of channels in the fungal cell membrane. The resultant loss of membrane permeability results in the loss of critically important molecules. Potassium ion efflux from the fungal cell and hydrogen ion influx cause internal acidification and a halt in enzymatic functions. Sugars and amino acids also eventually leak from an arrested cell. Fungistatic effects are most often evident at usual polyene concentrations. High drug concentrations and $\mathrm{pH}$ values 
between 6.0 and 7.3 in the surrounding medium may lead to fungicidal rather than fungistatic action (15).

\section{Nystatin}

In 1949, while conducting research at the Division of Laboratories and Research of the New York State Department of Health. It is derived from Streptomyces noursei (14). In 1955, Slome reported topical nystatin to be particularly effective for treatment of noninvasive candidiasis a frequent complication observed in children enrolled in early chemotherapeutic leukemia trials underway during this period $(15,16)$. Nystatin exhibited good activity against candida and modest activity against Aspergillus species. In aqueous solutions, nystatin forms aggregates that are toxic to mammalian cells both in vitro and in vivo. The insolubility and toxicity precluded its use as an intravenous therapy for systemic mycosis (17). Recently, a more soluble liposomal nystatin formulation (Nystran ${ }^{\circledR}$ ) with reduced toxicity was developed (18).The liposomal formulation consists of a freeze-dried, solid dispersion of nystatin mixed with a dispersing agent such as a poloxamer or polysorbate. The dispersing agent prevents aggregate formation in solution, increasing the drug's solubility and decreasing toxicity while maintaining efficacy (19). Liposomal nystatin has good activity in vitro against a variety of candida species including some amphotericin Bresistant isolate (15-19). Although the liposomal form of nystatin was less toxic than conventional nystatin, unacceptable infusion-related toxicity unfortunately caused a halt in the development of this drug (20).

\section{Amphotericin B}

Amphotericin B is the mainstay antifungal agent for treatment of life-threatening mycosis and for most other mycosis, with possible exception of the dermatophytosis. This drug binds to the membrane sterols of fungal cells, causing impairment of their barrier function and loss of cell constituents. Metabolic disruption and cell death are consequent upon membrane alterations. Investigations of the sterol content of mutant strains of Candida albicans and Cryptococcus neoformans has demonstrated that resistance is often associated with alterations in membrane sterol composition (7, 21, 22). Discovered by gold in 1956, it can truly be said represent a gold standards, it broad spectrum of activity includes most of the medically important moulds and yeasts, including dimorphic pathogens such as Coccidioides immitis, Histoplasma capsulatum, Blastomyces dermatitidis and Paracoccidioides brasiliensis. This antifungal agent of choice in treating most opportunistic mycoses caused by fungi such as Candida species, Cryptococcus neoformans, Aspergillus species and the zygomycetes (11-19) . Resistance to amphotericin B is rare, but is noteworthy for Pesudallescheria boydii, Fusarium spp, and Trichosporon spp. The drug must administered intravenously and is associated with numerous side effect, ranging from phlebitis at the infusion site and chills to renal toxicity, which may be severe. A major advance in the use of this agent has resulted from an understanding of the mechanism of its renal toxicity, which is presumed to involve tubuloglomerular feedback. The suppression of glomerular filtration could be reduced by administering sodium chloride (11-22).

\section{Natamycin}

Natamycin, also known as pimaricin and sometimes sold as Natacyn, is a naturally occurring antifungal agent produced during fermentation by the bacterium Streptomyces natalensis, commonly found in soil. Natamycin has a very low solubility in water, however, natamycin is effective at very low levels. There is an MIC (minimum inhibitory concentration) of less than 10 ppm for most molds. Natamycin is classified as a macrolide polyene antifungal and as a drug is used to treat fungal keratitis, 
an infection of the eye. This drug that natamycin acts via a novel mode of action and blocks fungal growth by binding specifically to ergosterol. It is especially effective against Aspergillus and Fusarium corneal infections (23).

\section{Azole}

The azole antifungal agents have fivemembered organic rings that contain either two or three nitrogen molecules, such as imidazoles or the triazoles respectively. The clinically useful Imidazoles are Clotrimazole, Miconazole and Ketoconazole. Two important triazoles are Itraconazole and Fluconazole. Over all the azole antifungal agents are thought to inhibit cytochrome $\mathrm{P}_{450}$-dependent enzyme involved in the biosynthesis of cell membrane sterols. Ketoconazole set the stage for the orally administered antifungal azoles $(24,25)$. It can be administered both orally and topically and has a range of activity including infections due to Histoplasma capsulatum and Blastomyces dermatitidis for which it is often used in nonimmunocompromised patients. It is also active against mucosal candidiasis and a variety of cutaneous mycosis, including dermatophyte infections, pityriasis versicolor and cutaneous candidiasis. It is not indicated for treatment of aspergillosis or systemic infections caused by yeasts. The triazoles such as Fluconazole, Itraconazole have become the standard for the azoles and have replaced amphotericin $B$ for managing certain forms of systemic mycosis. Fluconazole in new routinely used to treat candidemia in non-neutropenic host and is gaining acceptance for use in cryptococcosis and selected forms of coccidiomycosis. Itraconazole has proven to be effective for histoplasmosis, blastomycosis, sporotrichosis, coccidioidomycosis, consolidation treatment for cryptococcosis and certain and forms of aspergillosis. Fluconazole can be administered either orally or intravenously. Clinical isolates from diabetic patients had moderate resistance to fluconazole (FCZ). The lower sensitivity of Iranian isolates from Diabetes mellitus (DM) patients and their increased MIC patterns of antifungal agents may be related to the geography of the subject population, the environment of the lesion and microbial flora that exist in the lesions (26). The licensed formulation for Itraconazole is oral but, an intravenous formulation is under study and could be a significant addition directed at bioavailability problems relating to absorption of the oral formulation (25, 27). Side effect are not as common with the azoles as with amphotericin B, but lifethreatening liver toxicity can be arise with long-term use liver toxicity noted with ketoconazole has been problematic with the triazole. Other side effects include nausea and vomiting. Drug interactions are a potential problem between azoles and other drug classes and include cyclosporine, certain antihistamines, anticoagulants and antiseizure, oral hypoglycemic and other medications that are metabolized via similar pathways in the liver (27).

\section{Antimetabolites agent}

In contrast to the situation with antibacterial agents, few antimetabolites are available for use against fungi. The best example is 5-fluorocytosine, a fluorinated analog of cytosine. It inhibits both DNA and RNA synthesis via intracytoplasmic conversion to 5-fluororacil. The latter is converted to two active nucleotides: 5-fluorouridine triphosphate, which inhibits RNA processing and 5-fluorodeoxyuridine monophosphate, which inhibits thymidylate synthase and hence the formation of the deoxythymidine triphosphate needed for DNA synthesis. As with other antimetabolites, the emergence of drug resistance is a problem. Therefor 5fluorocytosine is seldom used alone. In combination with amphotericin $\mathrm{B}$ it remains the treatment of choice for cryptococcal meningitis is and effective against a number of other mycosis, including some caused by the dematiaceous 
fungi and perhaps even by Candida albicans(28-30).

\section{Other antifungal agents}

Griseofulvin is an antifungal antibiotic produced by Penicillium griseofulvum. It is active in vitro against most dermatophytes and has been the drug of choice for chronic infections caused by these fungi (e.g. nail infections with Trichophyton rubrum) since it is orally administered and presumably incorporated into actively growing tissue. It is still used in such instance but is being challenged by some of the newer azole antifungal agents (31). The drug inhibits mitosis and nucleic acid synthesis in fungi but has no effect against yeasts or other fungi. Griseofulvin is usually well tolerated. Adverse effects include headache, gastrointestinal disturbance and less commonly, urticarial, diarrhea and photosensitivity. The drug should be avoided during pregnancy and in patients with liver disease (32).

Potassium iodide given orally as a saturated suspension is uniquely used to treat cutaneous and lymphocutaneous sporotrichosis. This compound, interestingly is not active against sporothrix schenckii in vitro, it appears to act by enhancing the transepidermal elimination process in the infected host (5). Two other classes of antifungal agents represent new addition to topical treatment of the dermatomycosis in Europe. Two allylamines (naftifine, terbinafine) inhibit ergosterol synthesis at the level of squalene epoxidase; one morpholine derivative (amorolfine) inhibits at a subsequent step ergosterol pathway (33) (34).

\section{Future agents}

Powerful historical precedents support the use of antibody-based therapies to treat infectious diseases (35). However although still very early stages of development, newer approaches to the treatment of fungal infections will likely include the consideration of the host immune system and the interplay of drugs and host immunomodulators. Immunomodulators the therapies can be categorized as either pathogen specific or pathogen nonspecific (36). Pathogen-specific immunomodulators include antibody reagents and vaccines whereas cytokines, antimicrobial peptides and probiotics are considered pathogen nonspecific immunomodulators(37). Studies have shown immune sera to be protective in animal models of systemic candidiasis .Combination therapies using antifungal antibiotics with immunomodulators to treat invasive fungal disease are currently under investigation. To be of any clinical benefit, these regimens most improve efficacy without producing unacceptable side effects (3639). The immunodominant fungal antigen heat shock protein 90 (HSP90), expressed on cell surface of yeasts and certain malignant cells, has been investigated as a potential target for antibody therapy (40, 41).

Mycograb® (Neutec pharma, Antwerp, Belgium), a human recombinant monoclonal antibody against HSP90 was shown to have synergistic activity with amphotericin $\mathrm{B}$ in vitro against a broad spectrum of Candida species (42, 43). Mycograb ${ }^{\circledR}$ consist of an antigen-binding variable domain of heavy and light chains linked together to create a recombinant protein that can be expressed in Escherichia coli. The antifungal activity of this drug can be demonstrated using assays, such as minimal inhibitory concentration testing, used to assess conventional antifungal drugs $(43,44)$. Other new antifungal agent under study include naturally derived molecules with antifungal properties, such as the antifungal protein (AFP) secreted by Aspergillus giganteus. AFP is a small (94 amino acids) positively charged amphipathic protein that exert no cytotoxic or immunogenic effect on mammalian cell, but interferes with the physiological properties and synthesis of the fungal cell wall leading to fungal cell death $(45,46)$. Recently, Zumbuehl et al, reported that a 
new dextran-based hydrogel containing amphotericin B Prevented fungal infections for at least 53 days when implanted in mice. The history antifungal agents continues to evolve and no doubt will produce novel agents that, it is hoped will target the organism as well as the host immunity (47).

\section{Conclusion}

Progress has been in the development of new antifungal compounds or analogs of existing drugs with broad spectrum of activity, more favorable pharmacokinetic

\section{References}

1. Bennett JE, Dolin R, Blaser MJ. Principles and practice of infectious diseases: Elsevier Health Sciences; 2014.

2. Mowat E, Butcher J, Lang S, Williams C, Ramage G. Development of a simple model for studying the effects of antifungal agents on multicellular communities of Aspergillus fumigatus. J Med Microbiol. 2007;56(9):1205-12.

3. Richardson MD, Warnock DW. Fungal infection: diagnosis and management: John Wiley \& Sons; 2012.

4. Harvey JC, Cantrell JR, Fisher AM. Actinomycosis: its recognition and treatment. Ann Intern Med. 1957;46(5):868-85.

5. Urabe H, Nagashima T. Mechanism of antifungal action of potassium iodide on sporotrichosis. Int $\mathbf{J}$ Dermatol. 1969;8(1):36-9.

6. Shore RE, Moseson M, Xue X, Tse Y, Harley N, Pasternack BS. Skin cancer after X-ray treatment for scalp ringworm. Radiat Res. 2002;157(4):410-8.

7. Lattif AA, Swindell K. 1 History of Antifungals. Antifungal Therapy. 2016:1.

8. LAMB JH, Rebell G, Jones PE, Morgan RJ, Knox JM. Combined Therapy in Histoplasmosis and Coccidioidomycosis:

Methyltestosterone and Meth-Dia-Mer- profiles or better bioavailability. However, the development of more promising approaches as antifungal compounds with broader antifungal activity and fungalspecific mechanisms of action are a high priority.

\section{Acknowledgments}

The authors are extremely grateful to all specialists who have published valuable articles on antifungal medicines. Furthermore, we would like to thank Hamid Jafari for proofreading of the paper.

Sulfonamides. AMA archives of dermatology and syphilology. 1954;70(6):695-712.

9. Weed LA, Andersen HA, Good CA, Baggenstoss AH. Nocardiosis: clinical, bacteriologic and pathological aspects. N Engl J Med. 1955;253(26):1137-43.

10. Oxford AE, Raistrick H, Simonart P. Studies in the biochemistry of microorganisms: Griseofulvin, C17H17O6Cl, a metabolic product of Penicillium griseo-fulvum Dierckx. Biochem J. 1939;33(2):240.

11. Elewski BE. Mechanisms of action of systemic antifungal agents. J Am Acad Dermatol. 1993;28(5):S28-S34.

12. Odds FC, Brown AJ, Gow NA. Antifungal agents: mechanisms of action. Trends Microbiol. 2003;11(6):272-9.

13. Gentles J. Experimental ringworm in guinea pigs: oral treatment with griseofulvin. 1958.

14. Brown R, Hazen EL. Present knowledge of nystatin, an antifungal antibiotic. Transactions NY Acad Sci. 1957;19(5):447-56.

15. Ng A, Wasan KM, Lopez-Berestein G. Development of liposomal polyene antibiotics: an historical perspective. J Pharm Pharm Sci. 2003;6(1):67-83.

16. Silva L, Coutinho A, Fedorov A, Prieto M. Nystatin-induced lipid vesicles permeabilization is strongly dependent 
on sterol structure. Biochimica et Biophysica Acta (BBA)-Biomembranes. 2006;1758(4):452-9.

17. Sloane MB. A new antifungal antibiotic, mycostatin (nystatin), ior the treatment of moniliasis: a preliminary report. $\mathrm{J}$ Invest Dermatol. 1955;24(6):569-71.

18. Arikan S, Rex JH. Lipid-based Antifungal Agents Current Status. Curr Pharm Des. 2001;7(5):393-415.

19. Gupta AK, Sauder DN, Shear NH. Antifungal agents: an overview. Part II. J Am Acad Dermatol. 1994;30(6):91133.

20. Larson JL, Wallace TL, Tyl RW, Marr MC, Myers CB, Cossum PA. The reproductive and developmental toxicity of the antifungal drug Nyotran ${ }^{(l i p o s o m a l ~ n y s t a t i n) ~ i n ~ r a t s ~}$ and rabbits. Toxicol Sci. 2000;53(2):421-9.

21. Bekersky I, Fielding RM, Dressler DE, Lee JW, Buell DN, Walsh TJ. Pharmacokinetics, excretion, and mass balance of liposomal amphotericin B (AmBisome) and amphotericin B deoxycholate in humans. Antimicrob Agents Chemother. 2002;46(3):828-33.

22. Borisova MP, Brutyan RA, Ermishkin LN. Mechanism of anion-cation selectivity of amphotericin B channels. The Journal of membrane biology. 1986;90(1):13-20.

23. Te Welscher YM, Hendrik H, Balagué MM, Souza CM, Riezman H, De Kruijff $\mathrm{B}$, et al. Natamycin blocks fungal growth by binding specifically to ergosterol without permeabilizing the membrane. $\mathrm{J}$ Biol Chem. 2008;283(10):6393-401.

24. Como JA, Dismukes WE. Oral azole drugs as systemic antifungal therapy. $\mathrm{N}$ Engl J Med. 1994;330(4):263-72.

25. Holt RJ. Topical pharmacology of imidazole antifungals. J Cutan Pathol. 1976;3(1):45-59.

26. Raiesi O, Siavash M, Mohammadi F, Chabavizadeh J, Mahaki B, Maherolnaghsh M, et al. Frequency of Cutaneous Fungal Infections and Azole Resistance of the Isolates in Patients with Diabetes Mellitus. Advanced biomedical research. 2017;6.

27. Fromtling RA. Overview of medically important antifungal azole derivatives. Clin Microbiol Rev. 1988;1(2):187-217.

28. Haraguchi H, Taniguchi M, Motoba K, Shibata K, Oi S, Hashimoto K. Chrysodin, an antifungal antimetabolite. Agric Biol Chem. 1990;54(8):2167-8.

29. Arikan S, Rex J, Murray P, Baron E, Jorgensen J, Landry M, et al. Antifungal agents. Manual of clinical microbiology: Volume 2. 2006 (Ed. 9):1949-60.

30. Medoff G, Brajtburg J, Kobayashi G, Bolard J. Antifungal agents useful in therapy of systemic fungal infections. Annu Rev Pharmacol Toxicol. 1983;23(1):303-30.

31. Brian P. Studies on the biological activity of griseofulvin. Annals of Botany. 1949;13(49):59-77.

32. Napier EJ, Turner DI, Rhodes A. The in vitro action of griseofulvin against pathogenic fungi of plants. Annals of Botany. 1956;20(3):461-6.

33. Petranyi G, Ryder NS, Stutz A. Allylamine derivatives: new class of synthetic antifungal agents inhibiting fungal squalene epoxidase. Science. 1984;224(4654):1239-41.

34. Ryder NS, Mieth H. Allylamine antifungal drugs. Curr Top Med Mycol: Springer; 1992. p. 158-88.

35. Buchwald U, Pirofski L. Immune therapy for infectious diseases at the dawn of the 21st century: the past, present and future role of antibody therapy, therapeutic vaccination and biological response modifiers. Curr Pharm Des. 2003;9(12):945-68.

36. Heitman J. A fungal Achilles' heel. Science. 2005;309(5744):2175-6.

37. Casadevall A. The third age of antimicrobial therapy. Clin Infect Dis. 2006;42(10):1414-6.

38. Burnie JP, Carter TL, Hodgetts SJ, Matthews RC. Fungal heat-shock proteins in human disease. FEMS Microbiol Rev. 2006;30(1):53-88. 
39. Franco R, Pacheco R, Lluis C, Ahern GP, O'Connell PJ. The emergence of neurotransmitters as immune modulators. Trends Immunol. 2007;28(9):400-7.

40. Saito K, Dai Y, Ohtsuka K. Enhanced expression of heat shock proteins in gradually dying cells and their release from necrotically dead cells. Exp Cell Res. 2005;310(1):229-36.

41. Matthews R, Burnie J. Antibodies against Candida: potential therapeutics? Trends Microbiol. 1996;4(9):354-8.

42. Casadevall A. Antibody immunity and invasive fungal infections. Infect Immun. 1995;63(11):4211.

43. Pachl J, Svoboda P, Jacobs F, Vandewoude $\mathrm{K}$, van der Hoven $\mathrm{B}$, Spronk $\mathrm{P}$, et al. A randomized, blinded, multicenter trial of lipid-associated amphotericin B alone versus in combination with an antibody-based inhibitor of heat shock protein 90 in patients with invasive candidiasis. Clin Infect Dis. 2006;42(10):1404-13.

44. Matthews RC, Burnie JP. Recombinant antibodies: a natural partner in combinatorial antifungal therapy. Vaccine. 2004;22(7):865-71.

45. Meyer V. A small protein that fights fungi: AFP as a new promising antifungal agent of biotechnological value. Appl Microbiol Biotechnol. 2008;78(1):17-28.

46. Marx F, Binder U, Leiter E, Pocsi I. The Penicillium chrysogenum antifungal protein PAF, a promising tool for the development of new antifungal therapies and fungal cell biology studies. Cell Mol Life Sci. 2008;65(3):445-54.

47. Zumbuehl A, Ferreira L, Kuhn D, Astashkina A, Long L, Yeo Y, et al. Antifungal hydrogels. Proc Nation Acad Sci. 2007;104(32):12994-8. 